\title{
PROCESS AND SYSTEMS Scenario-based design for a hospital setting: An exploratory study of opportunities and barriers for personal health records usage
}

\author{
Authors: Christian P Subbe, ${ }^{A}$ Nick Pearson, ${ }^{B}$ Stephanie Wischhusen, ${ }^{B}$ Richard Hibbs, ${ }^{C}$ Sarah Wright ${ }^{D}$ \\ and Maria Xenou ${ }^{\mathrm{E}}$
}

Personal health records (PHRs) offer patients the opportunity to be more actively involved in their own care. There is limited research into the application during hospital admissions for elective or emergency presentations.

We used techniques from scenario-based design to test the opportunities and boundaries of a commercially available PHR in a simulated environment. Scenarios included a patient in his 80 s admitted for hip surgery with his son, and a younger patient admitted with pneumonia. A catastrophic deterioration was demonstrated with a mannequin in a highfidelity simulation. Workflows were summarised in swim-lane diagrams.

The PHR allowed patients to file information prior to the interaction with the clinical team. This led to shorter time requirements for acquisition of data. The elderly patient required assistance from a relative but this aided verification of history prior to the encounter with the clinical team. Ward rounds could be prepared by the patient with specific 'what matters' questions. Documentation in the PHR environment during a simulated life-threatening emergency did not result in information that was unintelligible or useful for the 'patient'.

Usage of a commercially available PHR during hospital admission is feasible and might aid workflow. Documentation of emergencies might require different documentation formats.

KEYWORDS: Health services research, personal health record, scenario-based design, crisis, inpatient

DOI: $10.7861 /$ fhj.2019-0061

Authors: ${ }^{A}$ consultant physician, senior clinical lecturer and improvement science fellow, Ysbyty Gwynedd, Bangor, UK, Bangor University, Bangor, UK and The Health Foundation, London, UK; ${ }^{B}$ foundation year doctor, Ysbyty Gwynedd, Bangor, UK; ' patient representative, Deganwy, UK; ${ }^{D}$ programme manager for Wales and lead for education, Patients Know Best, Cambridge, UK; E research and innovation lead, Patients Know Best, Cambridge, UK

\section{Background}

Hospital medicine is moving to paper light or paper free systems with the increasing use of electronic patient records. Patient care is moving away from the paternalistic approach to a shared decision-making model. Technological advances mean that patients are able to undertake increasing amounts of monitoring at home thus facilitating increased patient involvement by using personal health records (PHRs). PHRs are not a new concept but the methods of access are changing; patients are tracking their blood sugar via near field communication and smart phone apps rather than by recording finger prick records in a paper diary. ${ }^{1,2}$ Children have their personal child healthcare record (known as the 'red book'), traditionally a hard copy but now available online. ${ }^{3}$ Through the usage of PHRs, patients emphasised the importance of having 'a record of one's condition', being 'empowered to ask questions', and the potential for 'unwanted responsibility'.

There is a significant body of literature on PHRs, but this relates nearly exclusively to patients in primary care and chronic disease management programmes. ${ }^{5}$ Knowledge about safety impact of PHRs is largely limited to medication safety. There is only limited evidence for effects in hospital and none for usage in emergency care. ${ }^{6}$

Scenario-based design is a 'family of techniques in which the use of a future system is concretely described at an early point in the development process' ${ }^{7,8}$ It allows rapid interactive development of concepts and capture of possibilities and concerns.

We aimed to explore the opportunities for the usage of PHRs in hospital and explore the boundaries for patients to contribute to their own record through techniques of scenario-based design.

\section{Methods}

Study format

It was an observational feasibility study in a simulation laboratory using scenarios and role-play to explore boundaries of usage for the PHR.

\section{Setting}

The location was in the high-fidelity simulation laboratory at Ysbyty Gwynedd, Bangor. For the purpose of the study, Patients Know Best (PKB) provided a PHR in a digital test environment. 


\section{Participants}

Involved were medical doctors (foundation year-1 and foundation year-2), one physician associate, two student nurses and three patient actors (a woman in her 40 s and a man in his 80 s with his son).

\section{Intervention}

The intervention comprised use cases of common presentations for admission to hospital including elective and emergency admissions.

\section{Evaluation}

Participants of the workshop provided information on digital literacy and previous exposure to hospital admissions. The scenarios were recorded and analysed. Analysis included time and motion studies (ie time taken to perform documentation and related tasks) and semi-structured user feedback from patient actors, clinicians and students.

\section{Mapping process}

Graphic representation of processes using swim-lane diagrams to describe present workflow in local clinical practice were used, and possible future states as observed during the simulated scenarios.

\section{Patients Know Best properties}

Patients Know Best (PKB) allows patients and healthcare professionals to access medical records anytime, anywhere, and is controlled by patients who grant access to healthcare providers and hosted within the NHS N3 network. It is designed for use by individuals, NHS trusts, local authorities, charities, social enterprises and other organisations involved in the care of patients, particularly those with complex, long-term conditions necessitating multidisciplinary care from a plurality of providers. ${ }^{9}$ The platform connects health and care information from multiple primary and secondary care providers to create a single, unified copy of the data. Patients can then access their data via an online portal and use this to manage their health and wellbeing.

Patients can access and manage their appointments online, see test results with advice and explanations about what they mean, communicate directly with their healthcare professionals and seek medical advice in a timely way, share important information with nominated healthcare professionals, family members and/ or carers and link data from personal wearable devices to enhance monitoring.

PKB is the most deployed PHR system in the UK. As of October 2019, it has the records of 4 million patients in the UK and is used by researchers in eight European countries. PKB is the PHR supported by NHS Wales. ${ }^{10}$

\section{Scenarios}

Scenarios were repeated with variations to clinical course of the scenario and availability of digital resources (ie admission of a new patient with paper-based documentation vs admission of a new patient with electronic documentation provided by PKB). Timings of processes were derived during the scenarios and verified with the recording where required.

\section{Ethics and governance}

The study was approved by the Bangor Research Ethics Committee (REC reference 19/WA/0170). The patient actors gave informed consent, all clinicians and actors gave informed consent for the recording of the procedures.

\section{Results}

Four scenarios were examined; three used low-fidelity simulation and one used high-fidelity simulation.

\section{Scenario 1: Admission to hospital for elective hip replacement}

A patient in his 80 s presented with his son. Past medical history included atrial fibrillation on warfarin, constipation requiring regular laxatives (lactulose, occasional enemas), and an allergy to aspirin but had a stomach bleed after ibuprofen. The patient was concerned about pain in his hip. The pain had been present for 4 years, the patient had lost weight for improved mobility without success and needed regular pain killers. The patient would like to stay in hospital for as short a time as possible.

Collating the history took a significant amount of time. The patient had significant problems with arthritis and poor eyesight and was unable to operate the computer. His son was able to enter data for him and access information. There were clear discrepancies in the history documented by the son and details given by the patient. The presence of the son was felt to be essential to document reliable information.

During conventional history taking, time requirements from the team were 48 minutes ( 25 minutes for nursing and 23 minutes for medical history taking). During history taking using the PHR, time requirements for the team were 58 minutes (36 minutes for the patient, 10 minutes for nursing and 12 minutes for medical history taking).

\section{Scenario 2: Admission to hospital for an acute pneumonia}

A woman presented with cough, fever, sputum and abnormal vital signs. Past medical history included diabetes mellitus, hypertension, surgery for hiatus hernia, ex-smoker (used to smoke 20 cigarettes per day) and an allergy to co-trimoxazole. She is independent and likes baking cakes, has got several rescued cats and likes horse riding. Current medication include bendroflumethiazide, $2.5 \mathrm{mg}$ once per day (od), and ramipril, 10 mg od. The patient was worried to get back home and feed her cats. The patient had low oxygen saturations. A value was agreed, above which a transfer home would be usually safe.

The patient was able to enter all past medical history, medication and allergies as well as features of her acute illness into the platform. This led subjectively to a faster admissions process.

\section{Scenario 3: Next day ward round of the patient} from scenario 2

The patient was feeling better. The patient was able to access her vital-signs and these had improved in line with the criteria from the previous encounter. The patient remained worried to get back home and feed her cats. 
The patient decided to prepare the next day ward round online with questions to her care team about the diagnosis, the severity of her illness and the likely length of stay. This focused discussions during the acted ward round.

\section{Scenario 4: Peri-arrest post-hip-replacement}

High-fidelity simulation with a dummy (METIman, Medical Education Technologies) was used. The 'patient' was drowsy, had a low blood pressure and low respiratory rate.

The clinical team undertook an emergency assessment of airway, breathing, circulation, disability and exposure. Clinical symptoms were correctly identified as related to intra-operative opioids with hypoventilation. Usage of the electronic record during the peri-arrest situation was challenging; electronic documentation was slow, fields were on different screens and a high proportion of the documentation was medical jargon. Patient actors found it subsequently difficult to understand the meaning of the documentation.

\section{Participant feedback}

Scenarios were reviewed by the participants of the workshop. Swim-lane maps (Fig 1) were created to represent the present state from experience of the participants and compared with the process supported by the PHR.

Key observations were summarised by participants during debriefing.

> The elderly patient actor and his son could record and verify the past medical history and medication history prior the arrival of the medical team. This reduced the requirements for the 'clinicians' to be present during the process of negotiating the agreed version of truth. Using the electronic record resulted in prolonged periods of silence to find the correct data entry field and typing with interruption of the flow of conversation.

> Patients' entry of a comprehensive medical history takes a significant amount of time. By pre-populating data fields, this could be improved. A more focused system that captures only data pertinent to the current admission rather than comprehensive all system enquiries might reduce time requirements for documentation by healthcare professionals and improve workflow.

> A patient with the pneumonia was able to operate the system and complete large parts of her documentation without the assistance of a healthcare professional. The effects of this are captured in the swim-lane map. The patient actor felt empowered by seeing the same screen as the healthcare professional and having access to all information.

> Preparing for a ward round allowed the patient actor to ensure that areas of her interest were covered. Participants of the workshop felt that it was of advantage to have time prior to a ward round to focus on issues that were of importance to her. This included interest in the severity of the illness triggered by knowledge of the clinical diagnosis from the PHR. This observation is contrasted with clinical experience of patients complaining of having questions after the ward round or struggling to retain information about clinical diagnosis, treatment plan or planning of transfers of care.

> The clinical team was clearly overwhelmed by the usage of an unfamiliar electronic system to document clinical findings in real time during the simulated emergency. Due to the clinical requirements and under pressure, clinicians reverted to usage of jargon both in spoken and written communication. Design of shared records for inpatient usage would need to take this into account.

\section{Analysis of findings}

Timings and workflows were reviewed in the system with the PHR; patients and their carers, friends or family are able to perform some of the work that is traditionally part of the tasks undertaken by doctors or nurses. Access to records outside of the scheduled patient contact during admission and ward round allows patients time to articulate questions based on accessed information or their own ideas, concerns and expectations. This was also reflected in the subsequent feedback by the relative of the elderly patient actor (Box 1).

\section{Discussion}

In the present feasibility study, we have shown that patients could, in principle, engage in partaking in their own documentation even in an acute or emergency care setting. PHR usage allowed the patients, as well as the carer-actor, to add real value to the clinical information and verify important safety critical clinical information such as allergies, medication and previous complications.

Elderly patients are not only less technologically experienced but might additionally struggle with arthritis or visual impairment that might preclude direct interaction with a digital platform. At the same time, carers or family members might add measurable benefit if they are allowed to contribute their knowledge of the patient's condition.

The 'once for all' functionality of electronic records might reduce the strain not just on healthcare professionals but also on the patients who have to provide repeated answers to the same question in the current care system.

During a critical deterioration of a patient, electronic health records might be significantly slower and less agile than paperbased systems and the reliance on jargon to summarise complex safety critical information makes the documentation by clinicians nearly unusable for patients.

PHR can demonstrate value by providing a single view of a patient's history, creating one source of the truth, bringing together potentially divergent documentation from different sources to ensure all healthcare professionals have the right information at the right time to inform decision making, reduce duplication and enable a more preventative approach. Benefits of PHRs are obvious for many patients in chronic disease management programmes, such as inflammatory bowel disease, even if implementation and evaluation in the UK has been sporadic. ${ }^{5,11}$ A literature review of usage of patient portals in hospitalised patients found little evidence for studies that have formally evaluated the impact on clinical outcomes. ${ }^{6}$ Current designs might be problematic even in the more sheltered environment of an outpatient clinic and might require separate evaluation. ${ }^{12}$ Like others, we observed how electronic systems can aid to enhance communication between patients and healthcare professionals but might move focus from the patient to the screen and reduce eye contact. ${ }^{13}$

Recording of urgent information was slowed down by the electronic system. This is consistent with the literature; clinicians 
a

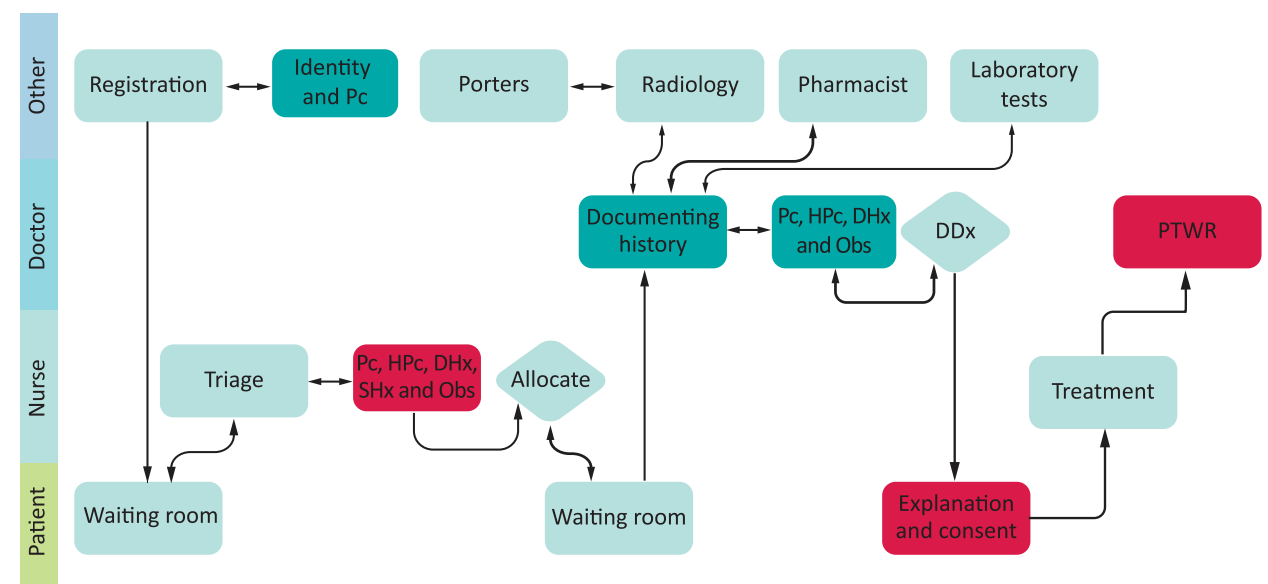

b

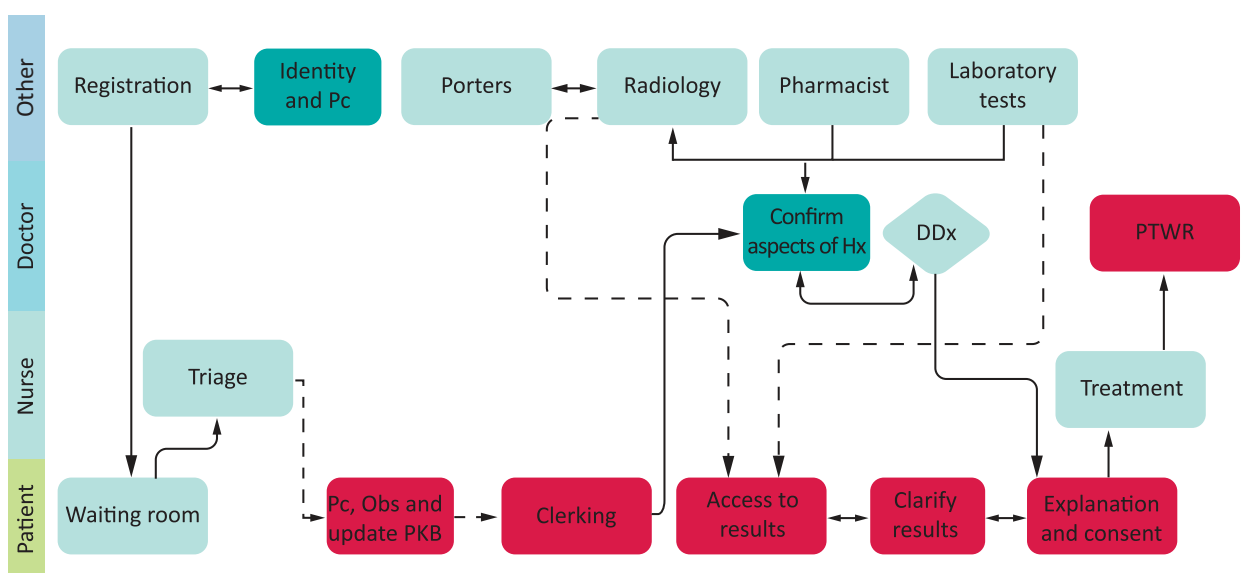

c

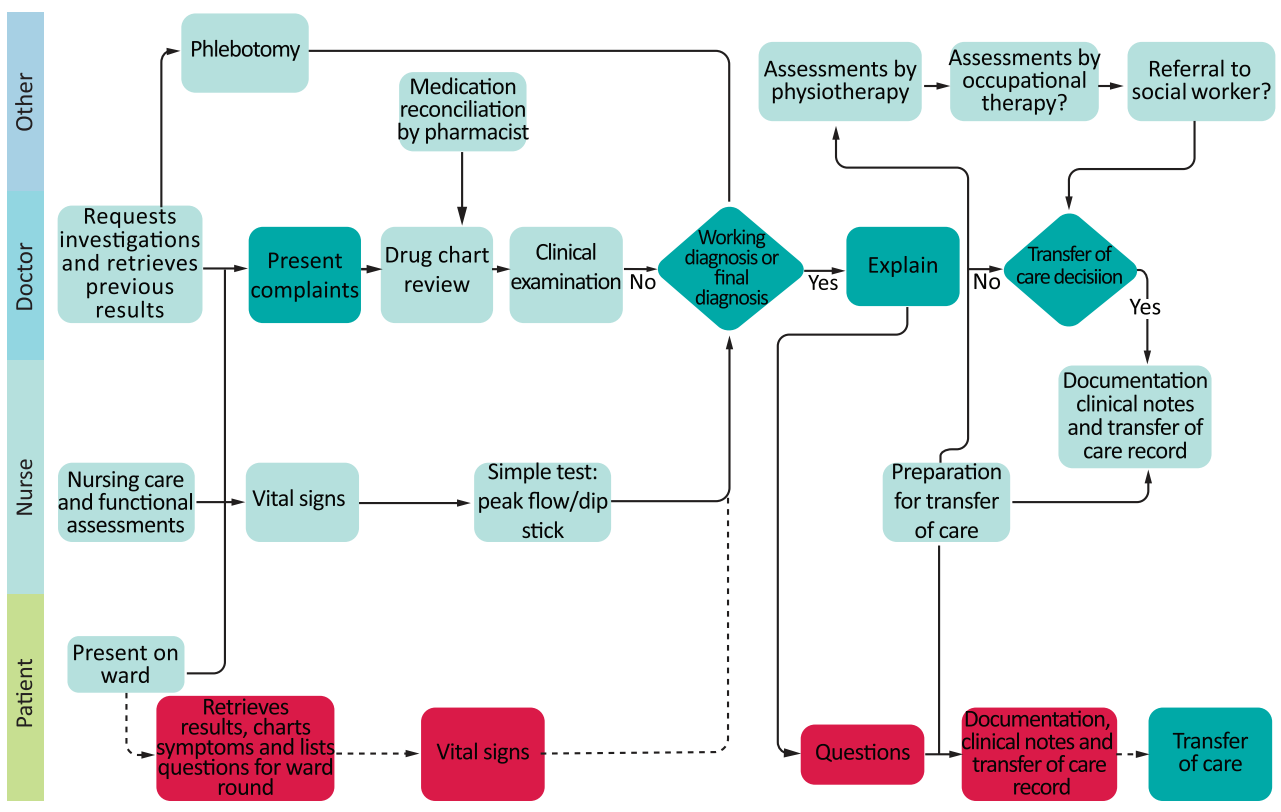

Fig 1. Swim-lane maps representing the experience of the participants currently compared with the process supported by the personal health record. a) Standard elective admission. b) Elective admission with personal health record. c) Ward round. DDx = differential diagnosis; $D H x=$ drug history; $\mathrm{HPC}=$ history of present complaint; Obs = observations; $\mathrm{Pc}=$ present complaint; $\mathrm{PKB}=$ Patients Know Best personal health record; $\mathrm{PTWR}=$ post-take ward round; $\mathrm{SHx}=$ social history. 


\section{Box 1. Observations by a relative}

Hospital admission and (emergency) triage represent a significant opportunity for the patient-carer dyad to explore and agree a common version of history; specifically those events and associated timeline leading to the present clinical episode. Basic disagreements over dates, the relevance of symptoms and the precision of recall must all be negotiated.

Data are generated within the dyad from multiple standpoints, not simply the primary perspectives of patient and carer but also those potentially arising from multiple roles played by the relative as carer, advocate and/or attorney, or indeed by the patient as both parent/child and sick person. The simulated elderly patient may wish to leave hospital as quickly as possible without too much attention being paid to their chronic constipation problem, whereas the simulated relative as carer may be more interested in a period of respite and a complete purge; while at the same time, as the patient's advocate or attorney, may consider that prolonged hospitalisation could result in further loss of independent living skills.

The personal health record (PHR) can facilitate the dyad in completing this information-gathering task precisely because it obliges both members to focus on achieving unanimity. The logical design inherent in any associated data collection tool(s) and underlying database systems can also assist in imposing the use of a common jargon-busting language on all participants, both data contributors (patients and carers) and all subsequent users (patients, healthcare practitioners and hospital administrators). However, some thought may need to be given to the fundamental unobservability of many variables of interest in the PHR (eg progress of disease) and that recording a single version of the truth may actually represent a loss of information, in particular the ability to assess degree of correlation between differing versions at the same timepoint.

Among the most important additional roles played by the carer when the PHR is computerised and the patient lacks dexterity, is visually impaired or simply does not belong to the digital era is that of the 'touchscreen operator' (cf medical scribe) controlling data input. While there may be few initial surprises (name, age, gender etc), the general influence of the touchscreen operator grows as time passes and symptom/medication lists and associated sub-menus become more interminable, which can result in an abbreviated approach to creating the record, whose ownership is then uncertain. The simulation was realistic in this respect.

perceive computer systems as slower than their previous practice and have implemented extensive workarounds including the usage of medical scribes. ${ }^{14}$ This has not changed over the last decade despite the advent of more advanced systems. ${ }^{15}$

To our knowledge, this is the first study that has describes the opportunities and boundaries of usage of PHRs in emergency and hyperacute care. The study was performed with a PHR from a single provider. This limits the ability to extrapolate the functionalities of other PHRs. We only examined a small number of scenarios but were able to capture typical hospital interactions. We did not address training requirements to fully use functionalities. For all users this was the first time they used a PHR and training might enhance usage and impact. ${ }^{16}$
We were unable to assess how patients would interact with the plethora of information generated by a modern hospital system and whether their attention would be drawn to mildly abnormal values rather than key clinical findings. We did not formally evaluate digital literacy or the impact of PHRs on activation. ${ }^{17,18}$ Satisfaction of patients and impact of records on clinical care might relate to these. ${ }^{19}$

PHRs have the potential to allow patients to feel a more active and valued member of the team. PHRs might facilitate time savings as patients can preload clinical records prior to being seen by healthcare professionals and reduce duplication. Current systems might not be optimised for usage in acute and emergency care. Given the high proportion of time that doctors are already spending on documentation, design and evaluation need to focus on using patient inputs in an efficient manner. ${ }^{20,21}$

Some of the benefits from usage of PHRs outside of hospital can be translated for inpatient usage. Development of tailored user interfaces for the specific tasks in acute care will require prospective testing in multicentre studies with capture of clinical outcomes, patient and staff satisfaction and cost implications. Platforms that are targeted at the ageing population might want to explore the usage of voice control or multi-user inputs that allow authorised friends and family members to support them.

\section{Conclusion}

PHRs have features that might usefully enhance care of patients admitted to hospital. Current systems might not allow them to share documentation of catastrophic deterioration in a meaningful way.

\section{Acknowledgements}

We are grateful for the generous support by the patient actors Neville Hibbs and Sara Williams (and Richard Hibbs, our co-author) who not only enabled the scenarios but gave detailed feedback of their experience which added to the depth of the results.

Bethan Mair Williams, Emma Austin and Rhonda Marie Griffin (and Nick Pearson and Stephanie Wischhusen, our co-authors) acted in nursing and medical roles in the scenarios. Leon Hughes and Einir Mowll from the team at the simulation laboratory at Ysbyty Gwynedd supported yet another research project in the most professional manner. Thank you!

\section{Conflicts of interest}

Christian Subbe is an improvement science fellow with The Health Foundation.

Patients Know Best (PKB) provided the personal health record test environment free of charge. Maria Xenou and Sarah Wright are members of the PKB team and were involved in the development of the study protocol and training of participants in the usage of the software.

\section{References}

1 Fischbach RL, Sionelo-Bayog A, Needle A, Delbanco TL. The patient and practitioner as co-authors of the medical record. Patient Couns Health Educ 1980;2:1-5.

2 Giglio R, Spears B, Rumpf D, Eddy N. Encouraging behavior changes by use of client-held health records. Med Care 1978;16:757-64. 
3 eRedbook: Personal child health record. eRedbook. www.eredbook. org.uk [Accessed 20 November 2019].

4 Sartain SA, Stressing S, Prieto J. Patients' views on the effectiveness of patient-held records: A systematic review and thematic synthesis of qualitative studies. Heal Expect 2015;18:2666-77.

5 Wyatt J, Sathanandam S, Rastall P, Hoogewerf J, Wooldridge D. Personal health record (PHR) landscape review: Final report. London: Royal College of Physicians, 2016.

6 Kelly MM, Coller RJ, Hoonakker PLT. Inpatient portals for hospitalized patients and caregivers: A systematic review. J Hosp Med 2017:13:405-12.

7 Carroll JM. Five reasons for scenario-based design. Interact Comput 2000;13:43-60.

8 Rosson MB, Carroll JM, Tech V, Va B. Scenario-based design. In: Jacko JA, Sears A (eds), The human-computer interaction handbook: Fundamentals, evolving technologies and emerging applications. L Erlbaum Associates, 2002:1032-50.

9 Davies P, Basu A, Safi A. Use of a web-based personal health record (Patients Know Best) to build closer relationships with people with Type 1 diabetes. Sandwell and West Birmingham Hospitals NHS Trust. www.patientsknowbest.com/sandwell-and-west-birminghamhospitals-nhs-trust.html [Accessed 07 September 2017]

10 Limb M. Patients in Wales to take control of medical records. BMJ 2017:357:j2982.

11 Carlsen K, Jakobsen C, Houen G et al. Self-managed eHealth disease monitoring in children and adolescents with inflammatory bowel disease: A randomized controlled trial. Inflamm Bowel Dis 2017;23:357-65

12 Yang Y, Asan O. Designing patient-facing health information technologies for the outpatient settings: A literature review. J Innov Heal informatics 2016;23:185.

13 Khoong EC, Cherian R, Matta GY et al. Perspectives of English, Chinese, and Spanish-speaking safety-net patients on clinician computer use: Qualitative analysis. J Med Internet Res 2019;21: e13131.

14 Shultz CG, Holmstrom HL. The use of medical scribes in health care settings: a systematic review and future directions. J Am Board Fam Med 2015;28:371-81.

15 Kaipio J, Hyppönen H, Lääveri T. Physicians' experiences on EHR usability: A time series from 2010, 2014 and 2017. Stud Health Technol Inform 2019;257:194-9.

16 McInnes DK, Solomon JL, Shimada SL et al. Development and evaluation of an internet and personal health record training program for low-income patients with HIV or hepatitis C. Med Care 2013;51(3 Suppl 1):S62-6.

17 Lobach DF, Hasselblad V, Wildemuth BM. Evaluation of a tool to categorize patients by reading literacy and computer skill to facilitate the computer-administered patient interview. AMIA Annu Symp Proc 2003;391-5.

18 Hibbard J, Helen G. Supporting people to manage their health: An introduction to patient activation. London: The King's Fund, 2014:51

19 Kelly MM, Coller RJ, Hoonakker PLT. Inpatient portals for hospitalized patients and caregivers: A systematic review. J Hosp Med 2018;13:405-12.

20 Sabin J, Subbe CP, Vaughan L, Dowdle R. Safety in numbers: lack of evidence to indicate the number of physicians needed to provide safe acute medical care. Clin Med 2014;14:462-7.

21 Sabin J, Khan W, Subbe CP et al. 'The time it takes...' How doctors spend their time admitting a patient during the acute medical take. Clin Med 2016;16:320-4.

Address for correspondence: Dr Christian Subbe, School of Medical Sciences, Bangor University, Bangor LL57 2DG, UK. Email: c.subbe@bangor.ac.uk

\section{Royal College} of Physicians

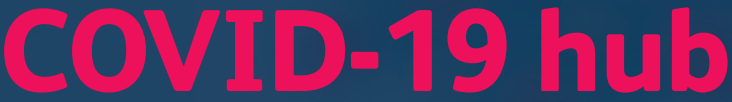

At the Royal College of Physicians we are committed to informing, supporting and advocating for our members who are at the heart of the COVID-19 pandemic and NHS response.

\section{Our COVID-19 online hub}

includes information on the latest developments for clinicians and links to up-to-date guidance.

Visit the hub at

www.rcplondon.ac.uk/covid-19 